\title{
Remembering the Beginnings
}

\author{
C.V. Negoita
}

\author{
Constantin Virgil Negoita \\ Computer Science, Hunter College, \\ City University of New York \\ http://www.hunter.cuny.edu/cs/Faculty/Negoita \\ E-mail: cnegoita@hunter.cuny.edu
}

\begin{abstract}
This paper wants to provide a personal view of the beginnings of fuzzy logic which was first introduced in the 1960's by Lotfi Zadeh, when fuzzy sets were presented as models of any vague concept, making possible the computation with words.
\end{abstract}

Keywords: fuzzy sets, fuzzy systems, vagueness

\section{Introduction}

We know Zadeh as we know no other eminent man who have made the last half of the last century memorable in science history. A mere glance at the materials to which we have access still suffice to show that our information regarding him is of such a kind as to leave scarcely anything to be desired. In the first place we have his papers. They are written with talent. He has not only left a minute record of his research during a space of nearly five decades but he found real pleasure in communicating on paper.

As a scientist, he was consistent. His memoir of himself remains unfortunately a fragment, but enough was completed to illustrate that portion of his career during which fuzzy sets were promoted. But if we owe much to the communicativeness of Zadeh himself, we owe much also to the communicativeness of his peers. The admiration of his followers is well known, but the picture which the critics drew of him was not always a pleasing one. They saw him not as he presented himself to the fascinated eye of friendship.

It should be remembered that they knew him only from a vivid immediacy that derives from the speech of witnesses, full of slang of the moment yet extremely serious because the fuzzy set seemed of the utmost cultural significance. What they painted was what they understood, and what they understood was very little.

\section{Fuzzy Sets and Generalized Sets}

It is common for logicians to give truth conditions for predicates in terms of set theory. "John is tall" is defined to be true just in case the individual John is in the set of tall men. Zadeh used the same path and said that "tall men" is a fuzzy set when the membership is a matter of degree.

In presenting the theory of fuzzy sets, we hoped to break through the bars of the prison of set theory. To understand a fuzzy set, imagine a two-dimensional world called Flatland. Each Flatlander is incarcerated in a flat set. We can peel him off and place him back somewhere else. If we fling a Flatlander into our three-dimensional world, he can see only two-dimensional cross sections of our world, a family of crisp sets. Simply put, by adding another dimension, we can capture more features. This is what a fuzzy set does. It adds a new dimension: our evaluation of the membership. Using classical flat mathematics, a fuzzy set can be represented by a family of crisp sets, projected on the Flatland. 
The classical rules of logic are represented by operations on the set with only two elements: true and false (0 and 1$)$.

In the universe of all sets - call it the category of sets - this important set is called classifier. When we tried to investigate the category of fuzzy sets, it was impossible to find a similar classifier. In fact in fuzzy set theory there is no fuzzy set of fuzzy subsets of a fuzzy set. The point is that there are two predicates in set theory: membership and equality. In the category of generalized sets, both can be fuzzy, but in fuzzy set theory only membership is allowed to be. This fact puzzled a lot of people.

Some critics bordered on the vituperative, and the tenets of fuzzy logic were dismissed as comical. Its arguments were declared frivolous and idle exercises in irrelevance and blasphemy.

In 1977 Arbib wrote bad reviews for the periodicals, and in 1984 Zeleny published a paper on the (ir)relevancy of fuzzy set theory. That, no doubt, explains why Herbert Toth, in 1987, in his $\mathrm{PhD}$ Thesis at the University of Vienna suspected that probably something has gone wrong in the development and interpretation of the theory.

Toth didn't deny Zadeh's original definition to be natural, immediate and elegant. This assertion was sufficiently justified by the vast amount of literature in an epidemically growing number of papers and books.

\section{$3 \quad$ Fuzzy Systems and the Postmodern Times}

Perhaps to deter us fuzzy people from further abuse, or perhaps only to improve our connection, Jim Bezdeck established our annual meetings. These are the origins of the international meetings that have today become pilgrimages, where all controversis were clarified when fuzzy sets were represented as families of crisp sets, and the Japanese started to implement fuzzy comptrollers, and, later, fuzzy systems were implemented as neural networks. But, still then, some mathematicians never understood fuzziness, because, for them, the precise specification of a set could be given only by binary logic.

The anatomy of a boom is simple. Over time, most ideas will rise in value. As this happens, people are attracted to them and this causes the ideas to rise more. This further gain attracts more people and gradually, perhaps over a period of a century, the number of people looking for this increase in value comes to determine what ideas are worth. The knowledgeable man, as he unwisely considers himself, is now concerned with the way an idea is attracting interest. That, rightly for the moment, determines its value.

The binary logic, beautiful, useful, and promising, determined the modern era, obsessed with mathematical models. Scientific truths became the pillars of progress. Zadeh, speaking of degrees of truth, shocked the foundation of modernity, and became the postmodern of information sciences. More than that, he defined postmodernity as a return to premodernity.

Why do postmodern scientists, in their advancing years, when love for precision survives, but only barely, cleave to the fluid vagueness as though it were cable for rappelling and not a tightrope any longer?

To embrace the whole from one point of view. The remarks made are less detailed, but more sure. You perceive each object less distinctly, but you describe the facts with more certainty. The details of the immense picture are lost in the shade, but you conceive a clear idea of the entire object.

In the philosophy of science this fact has been known for a long time. In the sixth century, Leontius from Bizantium observed that our impression of the world is vague, not revealing the details. If we attempt to particularize by division into species and individuals, the general view is lost: we are heading not towards truth but towards an infinite regress. In 1906, in France, Pierre Duhem, in a book about physics, its object and structure, distinguished between practical facts 
expressed in vague, ordinary language and theoretical facts expressed in precise, quantitative language. He argued that confidence in the truth of a vague assertion may be justified just because of its vagueness, which makes the assertion compatible with a whole range of observed facts. There is a balance between precision and certainty: one is increased only to the detriment of the other.

In Fuzzy Systems [1], a book I published in England, in 1981, I noted that one can build an infinite number of functions with values in an unit interval. In other words, we can say that a set never becomes exhausted in its evaluations. The set is massive, complete in itself, totally and wholly given, equivalent to the inert world of objects and things. As a result of an evaluation, a fuzzy set corresponds to the human consciousness. The set is both logically and ontologically prior to the fuzzy set. The latter is dependent upon the former. The fuzzy set is inconceivable without the set and is derived from it trough ordering. The fuzzy set is a borrowed being, an ordered set. It is through man that fuzziness enters the world. What is about the being of man that occasions fuzziness? The need of synthesis, the freedom to move up in the universe of concepts in order to find out stability. The reason that vague descriptions are used is to achieve stability.

Although awareness of the vagueness in our descriptions has existed for a very long time, only Zadeh's inquiries, which come under the heading "fuzzy", have brought matters to a head. It was Zadeh who coined the term when he spoke about fuzzy sets, blurred, indistinct in shape or outline, frayed, fluffy number of things of the same kind, together because they are similar, with different degrees of togetherness.

The description is fuzzy, not the things. People in the business of making exact descriptions concluded that an exact description is virtually impossible. This is a fact we have to accept and adjust to. Inexactness is not a liability. On the contrary, it is a blessing in the sufficient information it can convey with less effort. The vague description is easier to remember. That is inexactness makes for greater efficiency. Goguen in 1969 and Belman in 1970, agreed with Zadeh, when he said that precise quantitative analyses are not likely to have much relevance to the real-world problems which involve humans either as individuals or in gropus. He saw that as the complexity of a system increases, our ability to make precise and yet significant statements about its behavior diminishes. He saw all these things. He said also that precision and certainty are incompatible.

The 1960s were an age of innovation. At every corner critical thought and change were eating away the foundations of the traditional modern era.

In 1969 I defended a PHD thesis on Information Retrieval. To retrieve means to specify descriptors and rules for combining them. Immediately the question of vagueness came up. In my dissertation, I have questioned the meaning of vagueness in linguistic description with obsessive insistence. As my research went on, I began first to suspect, then to dislike, and finally to detest everything connected with boundaries between classes because a continuum exists that makes it impossible to do so satisfactorily.

In 1971 I published Information Storage and Retrieval which sounded another note of the famous war waged by common sense against the abuse of binary logic, a war in which so many were to engage. Out of this book grew Fuzzy Sets and their Applications, written together with Dan Ralescu in 1974, where we introduced the theorem of representation of a fuzzy set as a family of crisp sets.

\section{Some Final Remarks}

The history of fuzzy systems is marked by different visions. On the one hand a fuzzy set is a function. On the other hand a fuzzy set is a family of sets. The first vision took logic as its 
paradigm. The second vision made possible the recent embracing of connectionism.

For the idea of a model of vagueness, I was indebted to Zadeh whose paper on "Fuzzy Sets" appeared in 1965. It was he who first proved, it would seem, conclusively, that the meaning of words could be represented by numbers. To him we owe the first full and satisfactory representation of a vague word as a function.

Berkeley was the place where Zadeh was preaching his gospel, but his onslaughts were mercy compared with those terrible philippics in which, at Vanderbilt University in Nashville, Georgescu Roegen gave vent to his rage against arithmomorphism, the worship of numbers. In 1971, in an extraordinary book, The Entropy Law and the Economic Process, among the thesis that he defends is the claim that concepts are not arithmomorphic. They do not overlap. Concepts like "good" or "tall" have no boundaries. Instead they are surrounded by a penumbra within which they overlap with their opposites. At a particular historical moment, he notes, a nation may be both a democracy and a dictatorship just as there is an age when a man is both young and old. To the category of concepts we cannot apply the fundamental law of the binary logic, the principle of excluded middle (X cannot be both $\mathrm{A}$ and non- $\mathrm{A}$ ).

This axiom has so deeply imbued the modern era that it was felt to be natural and selfevident. Its denial seemed to be nonsensical. In opposition to the binary logic is what one might call premodern dogmatic logic, which assumes that A and non-A do not exclude each other as predicates. This logic was predominant in the first centuries. For the premodern Church Fathers, the opposition was a category of man's mind, not in itself an element of realty. The idea was that thought can only perceive in contradictions. The only way in which the world can be grasped ultimately lies, not in thought, but in the experience of oneness. (Three divine persons were grasped as one God).

Basically, fuzzy logic is based on the same feeling, and its applications allow engineers to create machines that approach human responses to stimuli, working with incomplete and unclear data to generate positive actions. Using fuzzy logic, Japanese washing machines are able to decide how dirty clothes are, how much water and soap should be used to wash them, and how long it should take to get them clean, all things an experienced launderer would know how to do instinctively. To me, the most impressive accomplishment was a fuzzy system built by Michio Sugeno, in 1985, at the Tokyo Institute of Technology, when he stabilized a helicopter that lost a rotor blade. No human pilot can manage that, and no mathematical model either.

Fuzzy systems, linguistically inspired, are a direct consequence of the seminal papers of Zadeh, published in the 1970s.

In 1975 classical fuzzy set theory had reached its apogee, since solutions of its basic problems were now at hand. Classical fuzzy set theory then changed from an heroic phase, in which we addressed ourselves to hitherto unfathomable questions, to an academic phase, in which a wealth of detail, albeit most important detail, was worked on by an army of competent scholars and technologists following well-established lines. The period of trail blazing was over, though most of the practical benefits were yet to be reaped.

\section{Bibliography}

[1] Negoita, C.V., Fuzzy Systems, Abacus Press, Tunbridge Wells, UK, 1981

[2] Negoita, C.V., Expert Systems and Fuzzy Systems, Benjamin Cummings, Menlopark, CA, 1985

[3] Negoita, C.V., Fuzzy Sets, Newfalcon Publications, Tempe, AZ, 2000 